\title{
Nearly Perfect Fluidity in the Dilute Fermi Gas: An Update
}

\author{
Thomas Schäfer
}

\author{
Department of Physics, North Carolina State University, Raleigh, NC 27695
}

\begin{abstract}
In this contribution we summarize recent results on the transport properties of strongly correlated dilute Fermi gases. We discuss the hydrodynamic equations in the normal phase and present new results on the structure of second order terms in the gradient expansion. We also discuss predictions from kinetic theory, and show how these predictions can be tested using experimental data on elliptic flow. We summarize current constraints on the shear viscosity to entropy density ratio $\eta / s$.
\end{abstract}

Keywords: quantum fluids, hydrodynamics, strong correlations.

PACS: $03.75 . S s, 05.60 . \mathrm{Gg}, 67.90 .+\mathrm{z}$.

\section{INTRODUCTION}

In recent years a remarkable convergence has occurred between novel physical properties of the hottest matter, the quark gluon plasma, and the coldest matter, cold atomic gases, that can be studied in the laboratory [1]. Both types of matter, which are governed by almost scale invariant interactions, were found to behave like nearly perfect liquids whose shear viscosity is close to the limits thought to be set by quantum mechanics. Initial estimates showed that in both the quark gluon plasma and the dilute Fermi gas at unitarity the ratio of shear viscosity to entropy density satisfies $\eta / s \lesssim 0.5 \hbar / k_{B}[2,3,4,5]$. This result is close to the value $\eta / s=\hbar /\left(4 \pi k_{B}\right)$ which is found in the strong coupling limit of a large class of field theories that can be analyzed using the AdS/CFT correspondence [6, 7]. This suggests that strongly coupled quark gluon plasmas and dilute Fermi gases are more efficiently described in terms of a dual gravitational theory than in terms of a conventional quasi-particle model.

In this contribution we summarize recent work on transport properties of dilute Fermi gases $[8,9]$. These systems have been realized experimentally using optically trapped alkali atoms such as ${ }^{6} \mathrm{Li}$ and ${ }^{40} \mathrm{~K}$. These two atoms are fermions because they posses a single valence electron and the nuclear spin is integer. At very low temperature the atoms can be described as pointlike particles interacting via interatomic potentials which depend on the hyperfine quantum numbers of the valence electron. A Feshbach resonance arises if a molecular bound state in a "closed" hyperfine channel crosses the threshold of an energetically lower "open" channel. Because the magnetic moments of the states in the open and closed channel are in general different, Feshbach resonances can be tuned using an applied magnetic field. At resonance the two-body scattering length in the open channel diverges, and the cross section $\sigma$ is limited only by unitarity, $\sigma(k)=4 \pi / k^{2}$ where $k$ is the relative momentum.

\section{HYDRODYNAMICS}

At large distances and long times the dynamics of the dilute Fermi gas is described by hydrodynamics. For simplicity we will only consider the normal phase, corresponding to $T>T_{c} \simeq 0.14 T_{F}$. Here, $T_{F}=k_{F}^{2} /(2 m)$ is the Fermi temperature, $k_{F}=\left(3 \pi^{2} n\right)^{2 / 3} /(2 m)$ is the Fermi momentum, and $n$ is the density. In the normal phase there are five hydrodynamic variables, the mass density $\rho=m n$, the flow velocity $\vec{v}$, and the entropy density $s$. These variables satisfy five hydrodynamic equations, the continuity equation, the Navier-Stokes equation, and the entropy production equation,

$$
\begin{aligned}
\frac{\partial \rho}{\partial t}+\vec{\nabla} \cdot(\rho \vec{v}) & =0, \\
\frac{\partial\left(\rho v_{i}\right)}{\partial t}+\nabla_{j} \Pi_{i j} & =0, \\
\frac{\partial s}{\partial t}+\nabla_{i j} j_{i}^{s} & =\frac{R}{T} .
\end{aligned}
$$


These equations close once we supply constitutive relations for the stress tensor $\Pi_{i j}$ and the entropy current $j_{i}^{s}$ as well as an equation of state. The constitutive relations can be determined order by order in gradients of the hydrodynamic variables. The stress tensor is given by

$$
\Pi_{i j}=\rho v_{i} v_{j}+P \delta_{i j}+\delta \Pi_{i j}
$$

where $\delta \Pi_{i j}$, the dissipative part, contains gradients of $v_{i}, \rho$ or $s$. At first order the dissipative contribution to the stress tensor is $\delta \Pi_{i j}=-\eta \sigma_{i j}$ with

$$
\sigma_{i j}=\left(\nabla_{i} v_{j}+\nabla_{j} v_{i}-\frac{2}{3} \delta_{i j}\left(\nabla_{k} v_{k}\right)\right)
$$

where $\eta$ is the shear viscosity and we have used the fact that the bulk viscosity of the unitary Fermi gas is zero [10]. The entropy current is $j_{i}^{s}=v_{i} s+\delta j_{i}^{s}$ with $\delta j_{i}^{s}=\kappa q_{i}$ and $q_{i}=\nabla_{i} \log (T)$. Here, $\kappa$ is the thermal conductivity. Finally, the dissipative function $R$ is given by $R=(\eta / 2) \sigma^{2}+\kappa q^{2}$. Scale invariance restricts the dependence of the shear viscosity and thermal conductivity on the density and the temperature. We can write $\eta(n, T)=\alpha_{n}(y) n$ and $\kappa(n, T)=\sigma_{n}(y) n / m$, where $\alpha_{n}(y)$ and $\sigma_{n}(y)$ are functions of $y=m T / n^{2 / 3}$.

We have recently studied the constraints imposed by Galilean invariance and conformal symmetry on the structure of second order terms in the gradient expansion [11]. These terms are potentially important because the leading order result given in equ. (5), which implies that the dissipative stresses are instantaneously determined by gradients of the velocity, can lead to acausal behavior [12]. We find that at second order

$$
\begin{aligned}
\delta \Pi_{i j}= & -\eta \sigma_{i j}+\eta \tau_{R}\left(\dot{\sigma}_{i j}+v^{k} \nabla_{k} \sigma_{i j}+\frac{2}{3}\left(\nabla^{k} v_{k}\right) \sigma_{i j}\right)+\lambda_{1} \sigma_{\langle i}{ }^{k} \sigma_{j\rangle k}+\lambda_{2} \sigma_{\langle i}{ }^{k} \Omega_{j\rangle k}+\lambda_{3} \Omega_{\langle i}{ }^{k} \Omega_{j\rangle k} \\
& +\gamma_{1} \nabla_{\langle i} T \nabla_{j\rangle} T+\gamma_{2} \nabla_{\langle i} P \nabla_{j\rangle} P+\gamma_{3} \nabla_{\langle i} T \nabla_{j\rangle} P+\gamma_{4} \nabla_{\langle i} \nabla_{j\rangle} T+\gamma_{5} \nabla_{\langle i} \nabla_{j\rangle} P,
\end{aligned}
$$

where $\tau_{R}, \lambda_{i}$ and $\gamma_{i}$ are second order transport coefficients, $\Omega_{i j}=\nabla_{i} v_{j}-\nabla_{j} v_{i}$ is the vorticity, and $\langle$.$\rangle denotes the$ symmetric traceless part of a second rank tensor. The most important part of this result is the structure of the terms proportional to $\tau_{R}$, which describe the relaxation of the dissipative stresses to the Navier-Stokes form. Conformal symmetry determines the particular linear combination of the comoving derivative of $\sigma_{i j}$ and $\left(\nabla^{k} v_{k}\right) \sigma_{i j}$ that appear in this term.

\section{KINETIC THEORY}

Near $T_{c}$ the transport coefficients $\eta$ and $\kappa$ are non-perturbative quantities that have to be extracted from experiment or computed in quantum Monte Carlo calculations. At high temperature (and at very low temperature, $T \ll T_{c}$, see [13]) transport coefficients can be computed in kinetic theory. The shear viscosity was first computed in [14]. Here we will follow the recent work [15] which also considers the frequency dependence of the shear viscosity. In kinetic theory the stress tensor is given by

$$
\Pi_{i j}=2 \int \frac{d^{3} p}{(2 \pi)^{3}} \frac{p^{i} p^{j}}{m} f_{p}
$$

where $f_{p}=f(t, x, p)$ is the distribution function of fermion quasi-particles and the factor 2 is the spin degeneracy. The equation of motion for $f_{p}$ is the Boltzmann equation. In order to extract the shear viscosity it is useful to consider the Boltzmann equation in a curved background metric $g_{i j}$. In this setting correlation functions of the stress tensor can be determined by computing variational derivatives with respect to the metric. The non-relativistic limit of the Boltzmann equation in a curved space with metric $g_{i j}$ is

$$
\left(\frac{\partial}{\partial t}+\frac{p^{i}}{m} \frac{\partial}{\partial x^{i}}-\left(g^{i l} \dot{g}_{l j} p^{j}+\Gamma_{j k}^{i} \frac{p^{j} p^{k}}{m}\right) \frac{\partial}{\partial p^{i}}\right) f(t, x, \mathbf{p})=C[f],
$$

where $\Gamma_{j k}^{i}$ is the Christoffel symbol and $C[f]$ is the collision integral. We consider small deviations from equilibrium and write $f=f_{0}+\delta f$ with $f_{0}(\mathbf{p})=f_{0}\left(p^{i} p^{j} g_{i j} /(2 m T)\right)$. We also write $g_{i j}=\delta_{i j}+h_{i j}$ and linearize in $h_{i j}$ and $\delta f$. We get

$$
\left(\frac{\partial}{\partial t}+\frac{p^{i}}{m} \frac{\partial}{\partial x^{i}}\right) \delta f+\frac{f_{0}\left(1-f_{0}\right)}{2 m T} p^{i} p^{j} \dot{h}_{i j}=C[\delta f] .
$$


This equation can be solved by going to Fourier space and making a suitable ansatz for $\delta f(\omega, k ; p)$. Once $\delta f$ is determined we can compute $\Pi_{i j}$ using equ. (7), and $\eta(\omega)$ by matching the $k \rightarrow 0$ limit to the hydrodynamic result equ. $(4,5)$. The result is

$$
\eta(\omega)=\frac{\eta(0)}{1+\omega^{2} \tau^{2}}, \quad \eta(0)=\frac{15(m T)^{3 / 2}}{32 \sqrt{\pi}}\left\{\begin{array}{cc}
1 & a \rightarrow \infty \\
1 /\left(3 m T a^{2}\right) & a \rightarrow 0
\end{array} .\right.
$$

We observe that the shear viscosity is large in the weak coupling limit $a \rightarrow 0$, and that $\eta$ decreases as the scattering length is increased. The shear viscosity saturates when the scattering length becomes comparable to the de Broglie wave length $a \sim \lambda_{d B} \sim(m T)^{-1 / 2}$. In this limit $\eta$ only depends on $\lambda_{d B}$ but not on the density or the scattering length. The frequency dependence of the shear viscosity is governed by the relaxation time $\tau=(3 \eta) /(2 \varepsilon)[16]$. We observe that relaxation is fast in the limit where the viscosity is small. We also observe that the viscosity satisfies a sum rule which only depends on thermodynamic quantities,

$$
\frac{1}{\pi} \int_{0}^{\Lambda} d \omega \eta(\omega)=\frac{\varepsilon}{3}
$$

where $\Lambda \sim T$ is a cutoff that takes into account the breakdown of kinetic theory at very high frequency. The sum rule can be extended to $\Lambda \rightarrow \infty$ by subtracting the high frequency tail the shear viscosity, $\eta(\omega) \rightarrow \eta(\omega)-C /(15 \pi \sqrt{m \omega})$, where $C$ is a temperature dependent constant known as the "contact" $[17,18,19,20]$.

There are several other interesting results that have recently been obtained in kinetic theory. Chao and Schäfer determined the second order coefficients defined in equ. (6) [11]. They find, in particular, that $\tau_{R}=(3 \eta) /(2 \varepsilon)$ agrees with the relaxation time in equ. (10). Braby et al. determined the thermal conductivity [21], and Bruun as well as Sommer et al. computed the spin diffusion constant [22, 23]. The spin diffusion constant at unitarity is

$$
D_{s}=\frac{9 \pi^{3 / 2}}{32 \sqrt{2} m}\left(\frac{T}{T_{F}}\right)^{3 / 2}
$$

This result can be compared to the momentum diffusion constant $D_{\eta}=\eta / \rho$. Kinetic theory predicts that $D_{\eta}=$ $45 \pi^{3 / 2} /(64 \sqrt{2} m)\left(T / T_{F}\right)^{3 / 2}$, which implies that the ratio of $D_{s} / D_{\eta}$ is independent of temperature. Near $T_{c}$, both diffusion constants are of order $\hbar / \mathrm{m}$. A similar relation holds in the quark gluon plasma, where the ratio of the heavy quark diffusion constant $D_{Q}$ and the momentum diffusion constant $D_{\eta}=\eta /(s T)$ is approximately constant [24].

\section{ELLIPTIC FLOW}

The first experiment that demonstrated nearly perfect fluidity in the dilute Fermi gas was the observation of elliptic flow by O'Hara et al. [25]. The experiment involves releasing the Fermi gas from a deformed, cylindrically symmetric, trap. The density evolves as

$$
n\left(x_{\perp}, x_{z}, t\right)=\frac{1}{b_{\perp}^{2}(t) b_{z}(t)} n_{0}\left(x_{\perp} b_{\perp}(t), x_{z} b_{z}(t)\right)
$$

where $x_{\perp}, x_{z}$ are the transverse and longitudinal coordinate, $b_{\perp}(t), b_{z}(t)$ are scale factors, and $n_{0}\left(x_{\perp}, x_{z}\right)$ is the equilibrium density of the trapped system. The initial system is strongly deformed, $A_{R}(0)=\left[\left\langle x_{\perp}^{2}\right\rangle /\left\langle x_{z}^{2}\right\rangle\right]^{1 / 2} \ll 1$. Hydrodynamic evolution converts the large transverse pressure gradient into transverse flow. As a consequence the aspect ratio $A_{R}(t)$ grows with time and eventually becomes larger than one, see Fig. 1.

Viscosity slows down the transverse expansion of the system. In order to quantify the effect of shear viscosity we have to solve the Navier-Stokes equation for the expanding cloud $[12,26]$. In general this has to be done numerically but for $\eta=\alpha_{n} n$ with $\alpha_{n} \simeq$ const very accurate semi-analytical scaling solutions can be found. For this purpose we make a linear ansatz for the force term $f_{i}=\left(\nabla_{i} P\right) / m=a_{i} x_{i}$ (no sum over $i$ ), where $a_{i}=a_{i}(t)$. The scale parameters $a_{i}$ and $b_{i}$ are determined by the coupled equations

$$
\begin{aligned}
\frac{\ddot{b}_{\perp}}{b_{\perp}} & =a_{\perp}-\frac{2 \beta \omega_{\perp}}{b_{\perp}^{2}}\left(\frac{\dot{b}_{\perp}}{b_{\perp}}-\frac{\dot{b}_{x}}{b_{x}}\right), \\
\frac{\ddot{b}_{z}}{b_{z}} & =a_{z}+\frac{4 \beta \lambda \omega_{z}}{b_{z}^{2}}\left(\frac{\dot{b}_{\perp}}{b_{\perp}}-\frac{\dot{b}_{z}}{b_{z}}\right),
\end{aligned}
$$



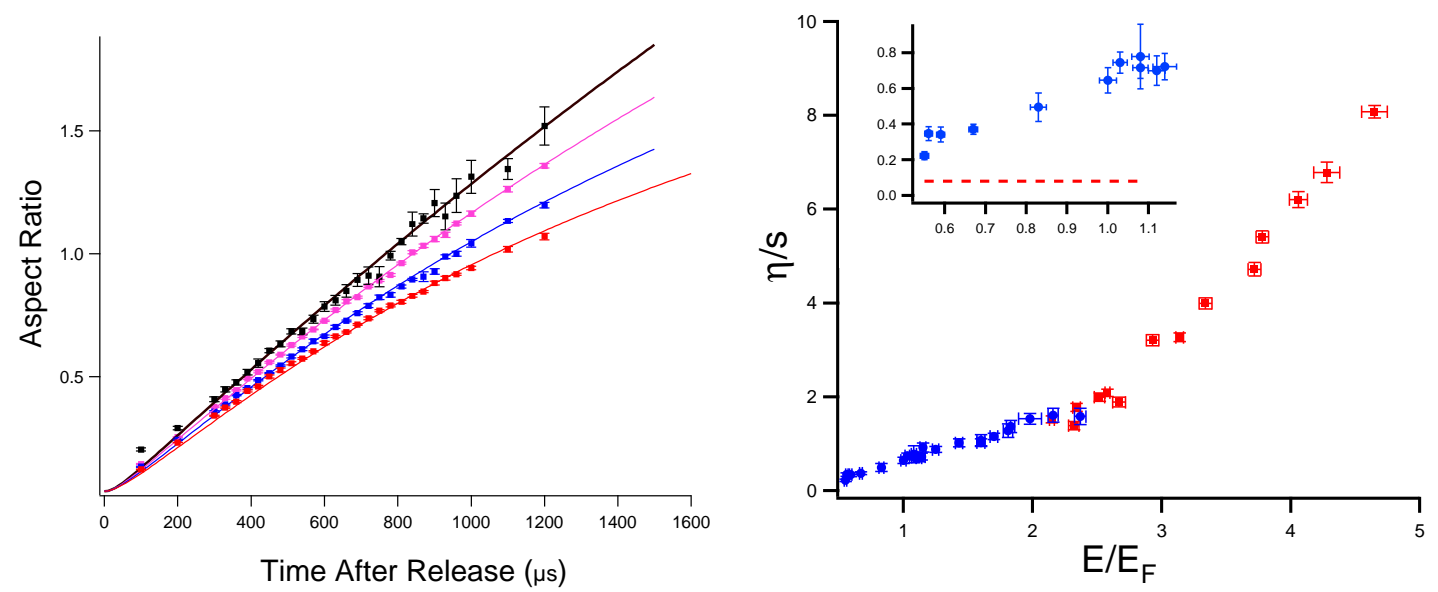

FIGURE 1. The left panel shows data for the aspect ratio versus time, from [27]: Top Black, $E=0.6 E_{F} ;$ Pink, $E=2.3 E_{F}$; Blue, $E=3.3 E_{F}$; Bottom Red, $E=4.6 E_{F}$. Solid curves: Hydrodynamic theory with the viscosity as the fit parameter. The panel on the right shows $\eta / s$ as a function of $E / E_{F}$. The red data points come from elliptic flow experiments, the blue data point were obtained from the damping of collective modes [27].

$$
\begin{aligned}
& \dot{a}_{\perp}=-\frac{2}{3} a_{\perp}\left(5 \frac{\dot{b}_{\perp}}{b_{\perp}}+\frac{\dot{b}_{z}}{b_{z}}\right)+\frac{8 \beta \omega_{\perp}^{2}}{3 b_{\perp}}\left(\frac{\dot{b}_{\perp}}{b_{\perp}}-\frac{\dot{b}_{z}}{b_{z}}\right)^{2}, \\
& \dot{a}_{z}=-\frac{2}{3} a_{z}\left(4 \frac{\dot{b}_{z}}{b_{z}}+2 \frac{\dot{b}_{\perp}}{b_{\perp}}\right)+\frac{8 \beta \lambda \omega_{z}}{3 b_{z}^{2}}\left(\frac{\dot{b}_{\perp}}{b_{\perp}}-\frac{\dot{b}_{z}}{b_{z}}\right)^{2},
\end{aligned}
$$

where $\omega_{\perp}, \omega_{z}$ are the oscillator frequencies of the harmonic confinement potential (before the gas is released). The parameter $\beta$ is defined by

$$
\beta=\frac{\alpha_{n}}{(3 N \lambda)^{1 / 3}} \frac{1}{\left(E_{0} / E_{F}\right)},
$$

where $N$ is the number of atoms, $\lambda=A_{R}(0)$ the initial aspect ratio, and $E_{0} / E_{F}$ the initial energy in units of $E_{F}=(3 N \lambda)^{1 / 3} N \omega_{\perp}$. The initial conditions are $b_{\perp}(0)=b_{z}(0)=1, \dot{b}_{\perp}(0)=\dot{b}_{z}(0)=0$, and $a_{\perp}(0)=\omega_{\perp}^{2}, a_{z}(0)=\omega_{z}^{2}$. Dissipative effects fall into two categories. The terms proportional to $\beta$ in equ. $(14,15)$ correspond to friction - shear viscosity slows down the expansion in the transverse direction. The dissipative terms in equ. $(16,17)$ describe reheating - shear viscosity converts some kinetic energy to heat which increases the pressure and eventually re-accelerates the system. Friction slows down the growth of $A_{R}(t)$ as compared to the ideal evolution. Reheating reduces this effect by about a factor of 2 .

In order to make comparisons with data we have to take into account that $\alpha_{n}$ is not a constant. In $[12,26]$ we argued that $\alpha_{n}$ in equ. (18) should be interpreted as the trap average of the local ratio of shear viscosity over density,

$$
\left\langle\alpha_{n}\right\rangle=\frac{1}{N} \int d^{3} x \alpha_{n}\left(\frac{m T}{n_{0}(x)^{2 / 3}}\right) n_{0}(x) .
$$

In the dilute corona of the cloud the local shear viscosity is independent of the density and equ. (19) is not well defined. This problem can be addressed by taking into account that the viscous stresses relax to the Navier-Stokes value on a time scale $\tau$ that becomes large as the density goes to zero, see equ. (10). A relaxation model for $\left\langle\alpha_{n}\right\rangle$ was studied in [12]. An even simpler model can be constructed based on the assumption that the shear viscosity relaxes to its equilibrium value at the center of the trap and is proportional to the density in the dilute corona. This implies that $\eta(x)=\eta(0)(n(x) / n(0))$. This parametrization agrees with the relaxation model at the $30 \%$ level. It was used by Cao et al. [27] to analyze the data shown in Fig. 1. The hydrodynamic curves shown in Fig. 1 were obtained with $\eta=\eta_{0}(m T)^{3 / 2}$ and $\eta_{0}=0.33$. This agrees quite well with the prediction of kinetic theory $\eta_{0}=15 /(32 \sqrt{\pi}) \simeq 0.26$, see equ. (10). Once we have verified the $T^{3 / 2}$ scaling behavior of the viscosity in the high temperature limit we can determine the shear viscosity to entropy density ratio all temperatures, see the right panel in Fig. 1. At very low temperature, $T \lesssim T_{F}$, dissipative effects in the elliptic flow experiment are very small and it is easier to determine the 
viscosity from the damping of collective modes, see the blue points in Fig. 1. We observe that the minimum of $\eta / s$ occurs near the lowest temperature studied, and that $(\eta / s)_{\min } \simeq 0.4$. We emphasize that these values are averaged over the trap, and that more detailed studies are needed to find the true minimum of $\eta / s$.

\section{CONCLUSIONS AND OUTLOOK}

There are a number of issues that need to be addressed before an accurate value of $\eta / s$ with fully controlled errors can be obtained. The most important of these is a better description of the transition from nearly perfect fluid dynamics in the center of the cloud to kinetic behavior in the dilute corona [28]. This problem can be addressed using the second order hydrodynamic equations discussed in this contribution. We would also like to develop tools that will allow us to perform calculations of transport properties in the strongly coupled regime. An important constraint for these calculations is provided by the sum rules presented in equ. (11). There have also been some very interesting attempts at extending the AdS/CFT correspondence to non-relativistic conformally invariant theories, see [29, 30]. Finally, we would like to understand whether nearly perfect fluidity in the dilute Fermi gas in the strongly interacting regime can be understood in terms of quasi-particle degrees of freedom. This question can be addressed by comparing different transport coefficients, like the ratio $D_{s} / D_{\eta}$ mentioned above, or by computing the spectral functions related to shear viscosity and diffusion.

Acknowledgments: This work was supported in parts by the US Department of Energy grant DE-FG02-03ER41260.

\section{REFERENCES}

1. T. Schäfer and D. Teaney, Rept. Prog. Phys. 72, 126001 (2009) [arXiv:0904.3107 [hep-ph]].

2. K. Dusling and D. Teaney, Phys. Rev. C 77, 034905 (2008) [arXiv:0710.5932 [nucl-th]].

3. P. Romatschke and U. Romatschke, Phys. Rev. Lett. 99, 172301 (2007) [arXiv:0706.1522 [nucl-th]].

4. T. Schäfer, Phys. Rev. A 76, 063618 (2007) [arXiv:cond-mat/0701251].

5. A. Turlapov, J. Kinast, B. Clancy, L. Luo, J. Joseph, J. E. Thomas, J. Low Temp. Phys. 150, 567 (2008) [arXiv:0707.2574].

6. G. Policastro, D. T. Son and A. O. Starinets, Phys. Rev. Lett. 87, 081601 (2001) [arXiv:hep-th/0104066].

7. P. Kovtun, D. T. Son and A. O. Starinets, Phys. Rev. Lett. 94, 111601 (2005) [arXiv:hep-th/0405231].

8. I. Bloch, J. Dalibard, W Zwerger, Rev. Mod. Phys. 80, 885 (2008) [arXiv:0704.2511].

9. S. Giorgini, L. P. Pitaevskii, S. Stringari, Rev. Mod. Phys. 801215 (2008) [arXiv:0706.3360].

10. D. T. Son, Phys. Rev. Lett. 98, 020604 (2007) [arXiv:cond-mat/0511721].

11. J. Chao, T. Schäfer, [arXiv:1108.4979 [hep-th]].

12. T. Schäfer and C. Chafin, Lecture Notes in Physics, in press (2010) arXiv:0912.4236 [cond-mat.quant-gas].

13. G. Rupak and T. Schäfer, Phys. Rev. A 76, 053607 (2007) [arXiv:0707.1520 [cond-mat.other]].

14. G. M. Bruun, H. Smith, Phys. Rev. A 72, 043605 (2005) [cond-mat/0504734].

15. M. Braby, J. Chao and T. Schäfer, New J. Phys. 13, 035014 (2011) [arXiv:1012.0219 [cond-mat.quant-gas]].

16. G. M. Bruun, H. Smith Phys. Rev. A 76, 045602 (2007) [arXiv:0709.1617].

17. E. Taylor and M. Randeria, Phys. Rev. A 81, 053610 (2010) [arXiv:1002.0869 [cond-mat.quant-gas]].

18. T. Enss, R. Haussmann and W. Zwerger, Annals Phys. 326, 770-796 (2011) [arXiv:1008.0007 [cond-mat.quant-gas]].

19. W. D. Goldberger, Z. U. Khandker, [arXiv:1107.1472 [cond-mat.stat-mech]].

20. J. Hofmann, [arXiv:1106.6035 [cond-mat.quant-gas]].

21. M. Braby, J. Chao and T. Schäfer, Phys. Rev. A82, 033619 (2010). [arXiv:1003.2601 [cond-mat.quant-gas]].

22. G. M. Bruun, New J. Phys. 13, 035005 (2011) [arXiv:1012.1607v2 [cond-mat.quant-gas]].

23. A. Sommer, M. Ku, G. Roati, M. W. Zwierlein, Nature 472, 201-204 (2011) [arXiv:1101.0780v1 [cond-mat.quant-gas]].

24. A. Adare et al. [PHENIX Collaboration], Phys. Rev. Lett. 98, 172301 (2007) [arXiv:nucl-ex/0611018].

25. K. M. O'Hara, S. L. Hemmer, M. E. Gehm, S. R. Granade, J. E. Thomas, Science 298, 2179 (2002) [cond-mat/0212463].

26. T. Schäfer, Phys. Rev. A, in press (2010) [arXiv:1008.3876 [cond-mat.quant-gas]].

27. C. Cao, E. Elliott, J. Joseph, H. Wu, J. Petricka, T. Schäfer, J. E. Thomas, Science 331, 58 (2011). [arXiv:1007.2625 [cond-mat.quant-gas]].

28. K. Dusling, T. Schäfer, Phys. Rev. A 84, 013622 (2011) [arXiv:1103.4869 [cond-mat.stat-mech]].

29. K. Balasubramanian and J. McGreevy, Phys. Rev. Lett. 101, 061601 (2008) [arXiv:0804.4053 [hep-th]].

30. D. T. Son, Phys. Rev. D 78, 046003 (2008) [arXiv:0804.3972 [hep-th]]. 